\title{
On Using Compressed Sensing for Efficient Transmission \& Storage of Electric Organ Discharge
}

\author{
Hussein Al-Azzawi ${ }^{1}$, Hong Huang ${ }^{1}$, Satyajayant Misra ${ }^{2}$, Wei Tang ${ }^{1}$ \\ 1 Klipsh School of Electrical and Computer Engineering, New Mexico State University \\ 2 Department of Computer Science, New Mexico State University \\ Email: azzawi@nmsu.edu, hhuang@nmsu.edu,misra@nmsu.edu,wtang@nmsu.edu
}

\begin{abstract}
In this paper, we present a compressed sensing based framework for a wireless sensor based biological sensing system for recording the frequency of fish electric organ discharge. We investigate the trade-offs between the parameters of compressed sensing, such as sampling matrix, reconstructed signal's signal to noise ratio, and compression ratio. The measured results show that our framework can be used to reduce the transmitted data size by $70 \%(3 x)$ while maintaining at least $10 \mathrm{~dB}$ signal to noise ratio for the reconstructed time domain frequency data. This size reduction with acceptable reconstructed signal quality will help reduce transmission energy and storage requirements for long-term sensing experiments, thus enabling the use of small and low power wireless sensors.
\end{abstract}

Keywords: Compressed sensing, wireless sensor networks, EOD.

\section{INTRODUCTION}

Research in biology requires the monitoring of events on the field and in the lab on a regular basis, which results in a large amounts of data being transported and stored. Wireless sensor networks (WSNs) have been used to enable such long-term and high-frequency collection of data. However, application of WSNs for monitoring of small animals, especially small fish and birds, presents unique challenges, which have been studied insufficiently. These challenges are: $i)$ need for small formfactor sensors $\left(1 \mathrm{~mm}^{3}\right.$ to $\left.2 \mathrm{~cm}^{3}\right)$, ii) need to sense and transmit data over long periods of time, resulting in transmission and storing of large amounts of data, and iii) need for ultra-low power consumption to prolong the life of the small battery on the sensor mote. We are currently in the design phase for the first challenge. However, this paper presents our results in the direction of addressing the last two challenges.

Motivation: This work emanates from our interdisciplinary research effort to instrument a biology lab with wireless sensors to record the electric organ discharge (EOD) of a mildly electric fish Sternopygus macrurus [1]. The electric field for the EOD of the $S$. macrurus is created by specialized cells called 'electrocytes.' Long term, automated, and scalable data collection systems are important tools for studying natural EOD differences among individuals as well as the variations in EOD due to behavioral or environmental factors. In prior research on the EOD of electric fish, the EOD signals have been recorded in live electric fish to study the effect of environmental factors and social interactions on the fish [2][4]. But, until our recent study [1], the largest study in the literature had accquired data for a maximum of one month. Figure. 1 shows an overview of the system we built [1].

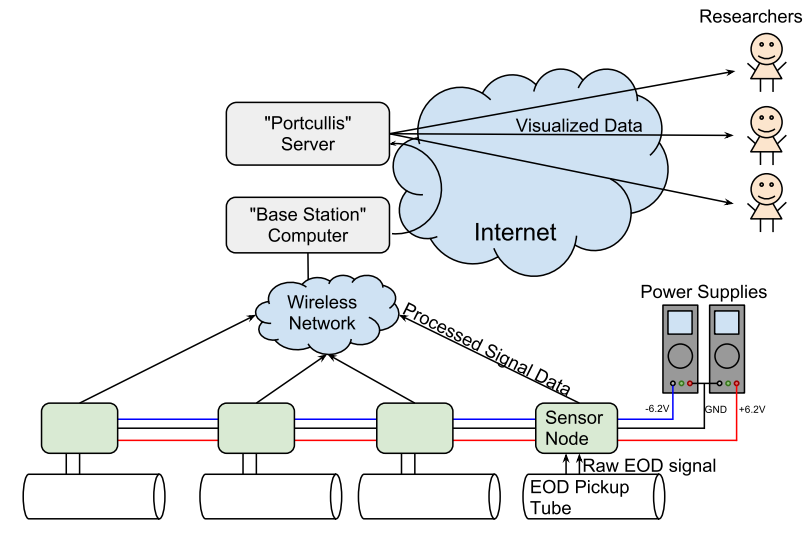

Fig. 1. System Overview.

The system consists of sensors to sense the EOD signals and transmit them over the wireless channel to a base station, which transmits the data to a cloud storage. The data can be accessed over the Internet and visualized remotely.

In the corresponding data gathering experiment on four fish in the lab, the data were gathered for more than six months, with the size of the gathered data quickly increasing to several hundred megabytes in a few days [1]. This especially brings to bear the challenges of having to transmit data over the wireless medium and store large amounts of data under the constraint of a strict power budget. The challenges became especially critical with the use of the miniature implanted wireless sensors.

Power consumption is the most important design parameter for wireless sensor nodes-especially in small miniature sensors. It is common knowledge that wireless sensor nodes consume the most power in wireless transmission, thus reducing power consumption during data transmission is an important point of focus. Reducing the number of bits transmitted has a direct impact on the reduction of power consumption-if the number of bits transmitted is halved the energy consumption can be reduced by more than half (the sensor can sleep more; no energy is consumed due to potential errors/retransmissions). This makes the technique of compressed sensing an attractive option [5].

Compressed sensing can be leveraged to compress the signal at the sender (sensor node) before transmission. At the receiver (base station), the original signal can be recovered with acceptable distortions. In this paper, we present our study 

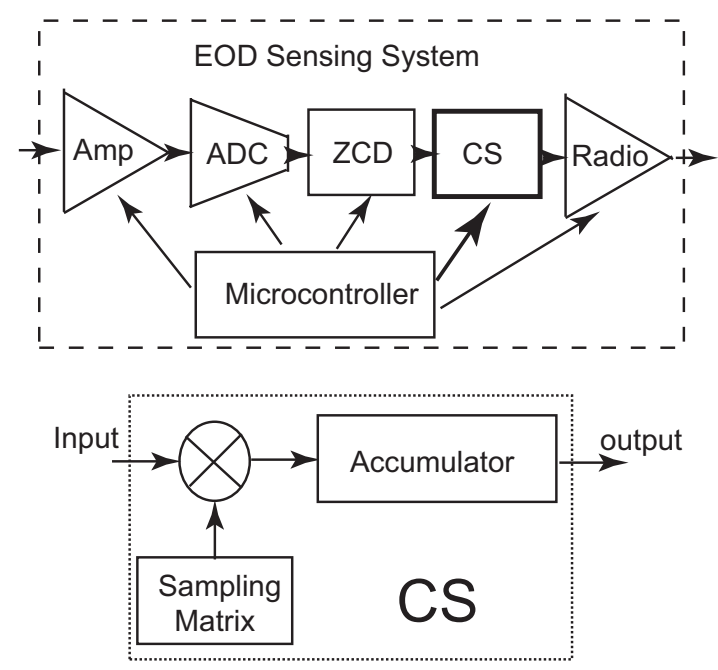

Fig. 2. Schematic diagram of the architecture of the EOD recording sensor.

on using compressed sensing to efficiently transmit the EOD signals of fish. We also detail the associated trade-offs among signal quality, compression ratio, and circuit complexity for our specific EOD signals. Compressed sensing has been used for reducing the transmitted data in the past, with sophisticated algorithms utilizing data-mining techniques used at the receiver to recover the signal [6]-[8]. However, there have been no study that has looked at using compressed sensing for transmission of the unique EOD signals on small fish, such as the $S$. macrurus.

Contributions: Our contributions in this paper are: (i) We present a novel framework that leverages compressed sensing to sense and transmit EOD signals. (ii) We demonstrate that by using our framework, the amount of data transmitted over the network can be reduced significantly, sometimes by as much as $70 \%$, with limited distortion when the signal is reconstructed at the receiver (SNR $\geq 10 \mathrm{~dB}$ ). Less data transmitted leads to less power consumption at the sensors. (iii) We also show how compressed sensing can be used to reduce long-term storage requirements for prolonged data gathering experiments that generate a large amount of data.

The rest of this paper is organized as follows. In Section II, we briefly provide the background on compressed sensing. In Section III, we describe our design that uses compressed sensing to collect EOD signals and evaluate the performance of our framework. The paper is concluded in Section IV.

\section{Compressed Sensing Background}

We provide the essential background on compressed sensing (CS) in this section. CS is highly effective in measuring signals that are sparse in a certain representation [9] [5]. A signal's sparsity is $k$ in a certain representation if the number of the signal's nonzero coefficients in that representation is $k$. Using $\mathrm{CS}$, we can recover the signal at a sampling rate far below the Nyquist rate, which is impossible using traditional techniques.

Consider an $n$-dimensional signal $f$, which is expressed in some orthonormal basis $\Psi=\left[\psi_{1} \psi_{2} \ldots \psi_{n}\right]$ as follows

$$
f=\sum_{i=1}^{n} x_{i} \psi_{i}=\Psi x
$$

where $x_{i}$ and $\psi_{i}$ are the $i$ th coefficient and $i$ th basis, respectively. According to CS theory, if $f$ is sparse in the basis $\Psi$, then, under certain conditions, taking $m$ measurements of $f$, where $m \ll n$, is sufficient to recover the signal exactly. Each measurement $y_{j}$ is a random projection of the original signal. The $m$-dimensional measurement can be represented by

$$
y=\Phi x
$$

where $y$ is the measurement vector and $\Phi$ is an $m \times n$ sensing matrix. To recover the signal, we solve the following minimization problem

$$
\min _{x}\|\Psi x\|_{1} \quad \text { subject to } \quad y=\Phi x .
$$

Good sensing matrices hold a property called restricted isometry property (RIP) [10] [11]. We define the isometry constant $\delta_{k}$ of a matrix $\Phi$ as the smallest number such that the following holds for all $k$-sparse vectors $x$

$$
\left(1-\delta_{k}\right)\|x\|_{2}^{2} \leq\|\Phi x\|_{2}^{2} \leq\left(1+\delta_{k}\right)\|x\|_{2}^{2} .
$$

Informally, we say a matrix $\Phi$ holds RIP of order $k$ if $\delta_{k}$ is not too close to one. The sensing matrices we use are two types of i.i.d Bernoulli binary random matrix $\mathrm{B} 1$ and $\mathrm{B} 2$ given below:

B1: $\operatorname{Prob}\left(\phi_{i, j}=1 / \sqrt{m}\right)=p, \operatorname{Prob}\left(\phi_{i, j}=-1 / \sqrt{m}\right)=1-p$
B2: $\operatorname{Prob}\left(\phi_{i, j}=1 / \sqrt{m}\right)=p, \operatorname{Prob}\left(\phi_{i, j}=0\right)=1-p$

where $p$ is the Bernoulli probability. Both $\mathrm{B} 1$ and $\mathrm{B} 2$ random matrices hold RIP with high probability when $p$ is not too close to zero or one. Good signal recovery is ensured if the number of measurements $m$ satisfies the following inequality,

$$
m \geq c k \log (n / k)
$$

where $c$ is some constant dependent on the particular sensing matrix used, and $k$ is the sparsity of the signal [10] [11]. We use $l_{1}$-MAGIC [12] to recover signal, which is a frequently used software package for solving CS problems. $l_{1}$-MAGIC solves the convex optimization problem (i.e. Equation (2)) in CS using the standard interior-point method.

\section{Compressed Sensing in EOD Recording}

Figure 2 illustrates the architecture of our EOD recording system. The analog input signal is amplified and converted into a digital stream. A Zero Crossing Detector (ZCD) calculates the frequency of the input signal during a predefined time period. In our previous system, the frequency data were sent to the radio directly [1]. In this framework, our proposed CS system is inserted between the ZCD and the radio. A microcontroller controls parameters of each building block. It is able to control the compression ratio and sampling matrix of the CS building block. 
Tank 4|Frequency

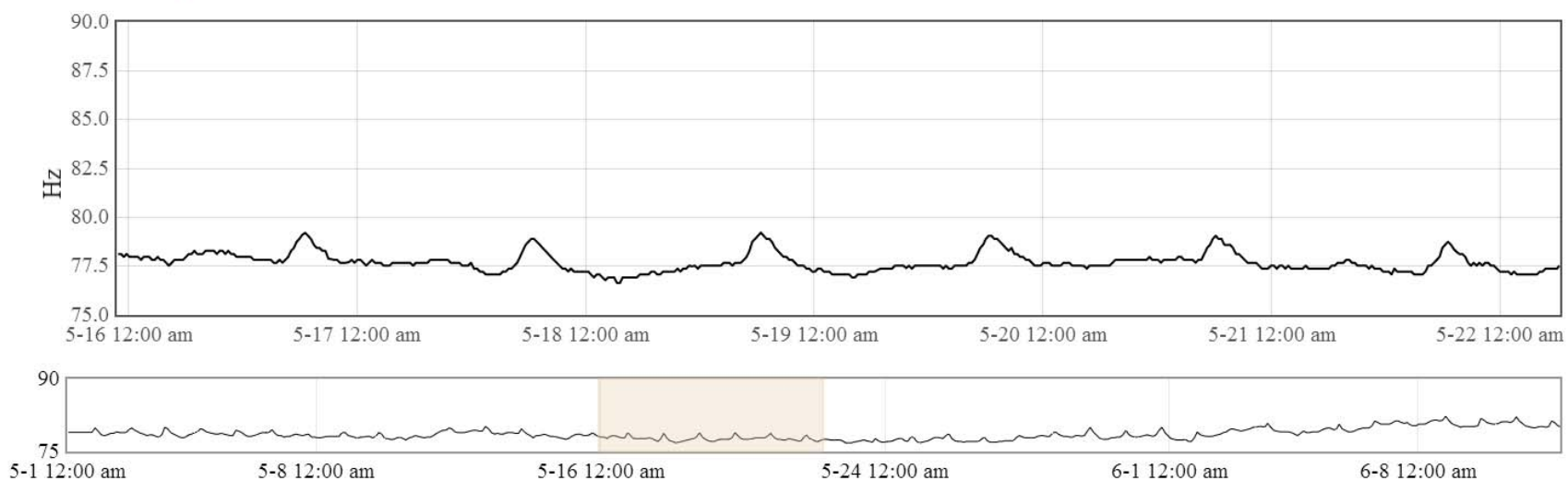

Fig. 3. A 40 days snapshot of the EOD signal of the fish in Tank 4 with a zoomed-in portion from 5/16/2013 to 5/22/2013.

The raw frequency data from the ZCD is the input of the CS block. A pre-defined random matrix will be mixed with the raw data, and the result is fed into an accumulator. The output of the accumulator becomes the measurement vector $y$.

Figure 3 shows a 40 days snapshot of the EOD signal captured from one of the four fish (fish in Tank 4) during our long term data collection. The graph on top shows a zoomedin portion (5/16/2013-5/23/2013). The figure clearly shows a pattern with the frequency rising and falling periodically at the same time during the day. This frequency pattern suggests a correlation between the frequency of the EOD of a fish and its circadian rhythm. We note that biologists are particularly interested in the frequency of the EOD signal. Hence unlike other CS applications which record analog signals [6]-[8], in the EOD sensing system we record the time domain frequency data. Similar to EMG waveforms [7], the EOD waveform is also sparse in both time and frequency domain. In this work, we apply CS in the time domain to reduce the recorded data size.

We used the Bernoulli matrices B1 and B2 defined in Equations (5) and (6) as the sampling matrices to simplify the hardware, which we will implement in the future. We investigated the relationship between the compression ratio, $\rho$, and the reconstructed signal quality for the EOD signal. The compression ratio $\rho$ is equal to $m / n$, where $n$ is the number of original data points and $m$ is the number of data points in the corresponding compressed signal. The compression ratio $\rho$ is an indicator of how much reduction in data transmission and storage we can achieve using CS, and it is also an indicator of how much reduction in power consumption we can achieve. We use the signal to noise ratio $(S N R)$ to evaluated the reconstructed signal's quality. The SNR is given by,

$$
S N R=10 \cdot \log _{10} \sum_{i=1}^{n} \frac{S_{i}^{2}}{\left(\hat{S}_{i}-S_{i}\right)^{2}}
$$

where the noise is defined by the difference between the original signal $(S)$ and the reconstructed signal $(\hat{S})$. The relationship between $\rho$ and the SNR depends on the actual signal sparsity and the sampling matrix. Our goal is to seek the highest possible signal compression ratio, i.e. lowest possible
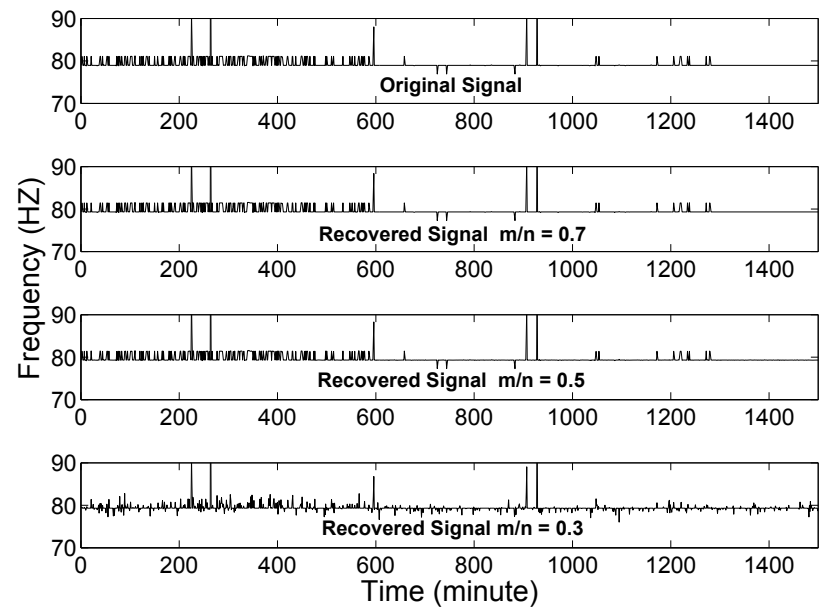

Fig. 4. EOD frequency signal and the compressed sensing reconstruction for different $\rho=m / n$, using B1 matrix.

value of $\rho$, while satisfying the constraint that the SNR of the reconstructed signal be at least $10 \mathrm{~dB}$. The $10 \mathrm{~dB}$ SNR value is commonly used in the literature as a quality threshold [7].

Figure 4 illustrates a portion of the CS EOD recording of one of the fish and the corresponding reconstructed signal with different compression ratios. The number of data points here are $n=1500$. Note that as the compression ratio $\rho=m / n$ decreases, the reconstructed signal has more artifact and the SNR decreases. However, it is noteworthy that even when $\rho=$ 0.3 , the primary frequency values are still recoverable with fairly high degree of accuracy. Figure 5 shows the SNR as a function of the compression ratio $\rho$ when the two matrices B1 and $\mathrm{B} 2$ are used. For EOD frequency signal, the SNR drops to $10 \mathrm{~dB}$ when the compression ratio is 0.3 . This implies that with the compressed signal being as much as $30 \%$ of the size of the original signal, the original signal can still be accurately reconstructed. This also means that the compressed signal can be stored instead of the original signal, to be reconstructed later when needed, thus reducing the storage requirements by $70 \%$. Figure 5 also shows that the SNR is higher when B1 matrix is applied than when B2 is applied, which is a well known fact in the CS field. But this involves a tradeoff between 


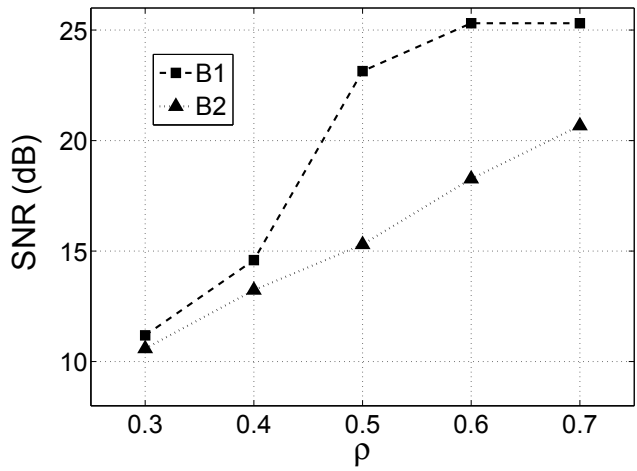

Fig. 5. SNR of the reconstructed signal for different values of $\rho(n=1500)$.

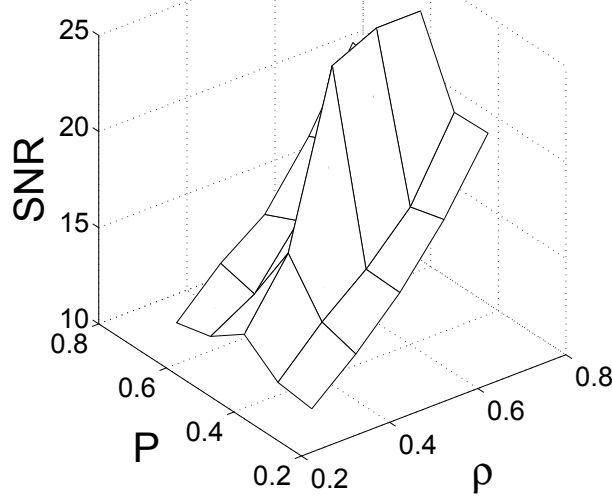

Fig. 6. 3-D plot showing the relationship among SNR, $\rho$, and $p$ (B1 matrix).

the SNR and the measurement cost. B2 matrix incurs less measurement cost than B1 matrix because 0's in B2 means no measurement.

In the sampling matrices $\mathrm{B} 1$ and $\mathrm{B} 2, p$ represents the probability that an element of the matrix is +1 . Figure 6 shows the relationship between SNR, the compression ratio $\rho$, and $p$ for the sampling matrix B1. An interesting observation is that the SNR is maximum for $p=0.5$ and and $\rho>0.6$. The SNR is strongly dependent on the value of $p$, dropping sharply when $p$ deviates from 0.5 . Thus $p=0.5$ is a desirable value as it results in higher SNR. Another interesting observation is that for $p=0.5$ the decline in the SNR is gradual with the decrease in $\rho$. We observe similar phenomena when B2 matrix is used.

We applied our CS algorithm on data from two different fishes in Tanks 2 (high frequency) and 4 (low frequency). Figure 7 shows the compressed and reconstructed waveforms for the high frequency and the low frequency EOD signals respectively $(\rho=0.5)$. It is also demonstrated that CS performs consistently for different signals.

\section{COnclusions}

In this paper, we proposed a novel compressed sensing based framework for EOD frequency signal sensing. We investigated the relationship between the system parameters such as compression ratio, the SNR and different sampling matrices. The results show that by using compressed sensing

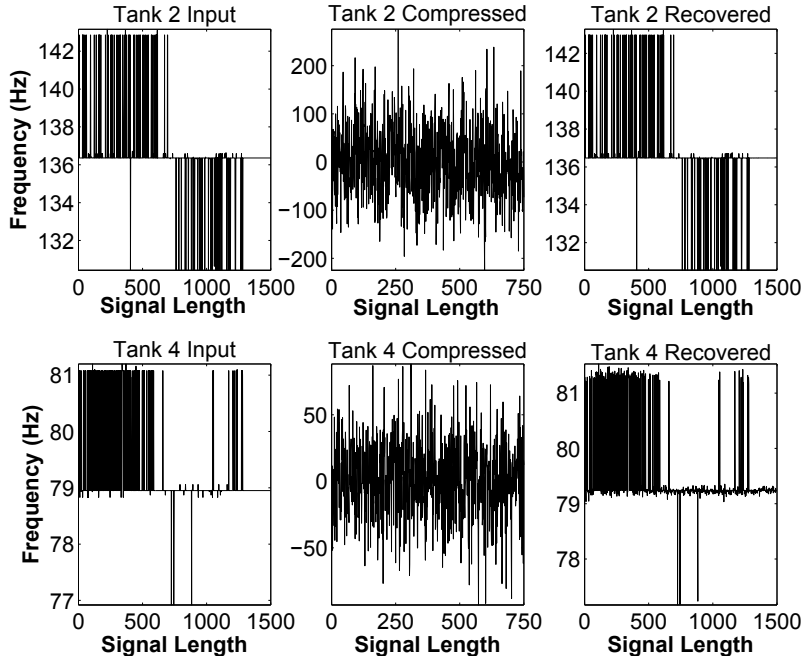

Fig. 7. Compressed sensing waveform from two EOD tanks.

the data size can be reduced by $3 \mathrm{x}$ without losing significant amount of information.

\section{ACKNOWLEDGement}

This work is funded by the U.S. National Science Foundation (NSF) Grant CNS-1248109.

\section{REFERENCES}

[1] M. Harris, E. Salazar, R. Gueth, V. Nawathe, M. Sharifi, W. Tang, and S. Misra, "Wireless sensing framework for long-term measurements of electric organ discharge," in IEEE Biomedical Circuit and Systems Conference, 2013.

[2] P. Stoddard, M. Markham, V. Salazar, and S. Allee, "Circadian rhythms in electric waveform structure and rate in the electric fish brachyhypopomus pinnicaudatus," Physiology \& Behavior, vol. 90, no. 1, pp. 11-20, 2007.

[3] C. Franchina and P. Stoddard, "Plasticity of the electric organ discharge waveform of the electric fish brachyhypopomus pinnicaudatus i. quantification of day-night changes," Journal of Comparative Physiology A, vol. 183 , no. 6, pp. 759-768, 1998.

[4] C. Franchina, V. Salazar, C. Volmar, and P. Stoddard, "Plasticity of the electric organ discharge waveform of male brachyhypopomus pinnicaudatus. ii. social effects," Journal of Comparative Physiology A, vol. 187, no. 1 , pp. $45-52,2001$.

[5] D. Donoho, "Compressed Sensing," IEEE Transation on Information Theory, vol. 52, no. 4, pp. 1289-1306, 2006.

[6] M. Shoaran, M. Lopez, V. Pasupureddi, Y. Leblebici, and A. Schmid, "A low-power area-efficient compressive sensing approach for multichannel neural recording," in IEEE International Symposium on Circuits and Systems (ISCAS), 2013, pp. 2191-2194.

[7] K. Wilhelm and Y. Massoud, "Compressive sensing based classification of intramuscular electromyographic signals," in IEEE International Symposium on Circuits and Systems (ISCAS), 2012, pp. 273-276.

[8] J. Zhang, Y. Suo, S. Mitra, S. Chin, T. Tran, F. Yazicioglu, and R. Etienne-Cummings, "Reconstruction of neural action potentials using signal dependent sparse representations," in IEEE International Symposium on Circuits and Systems (ISCAS), 2013, pp. 1520-1523.

[9] J. R. E. Candes and T. Tao, "Robust uncertainty principles: Exact signal reconstruction from highly incomplete Fourier information," IEEE Transation on Information Theory, vol. 52, no. 2, pp. 489-509, 2006.

[10] E. Candes and T. Tao, "Decoding by linear programming," IEEE Transation on Information Theory, vol. 51, no. 12, pp. 4203-4215, 2005.

[11] J. Tropp, "Greed is good: Algorithmic results for sparse approximation," IEEE Transation on Information Theory, vol. 50, no. 10, pp. 2231-2242, 2004.

[12] “ $l_{1}$-magic," http://users.ece.gatech.edu/ justin/11magic. 